\title{
Note on the Stabilization of Acetylglycosyl Halides and Sugar Acetates
}

\section{By Horace S. Isbell and Harriet L. Frush}

In connection with an investigation of the stereomeric factors affecting replacement reac. tions, ${ }^{1}$ it was necessary to prepare and store a number of acetylglycosyl bromides. These compounds are useful intermediates for the synthesis of sugars and many sugar derivatives. Their utility for synthetic purposes depends upon the high reactivity of the halogen, but this property also makes them relatively unstable. Decomposition is accelerated by the presence of water, alcohol, acids, or other substances that react with active halogen; the resultant hydrogen halide, unless removed, acts catalytically to cause further deterioration. To restrict decomposition, it has been customary to store acetylglycosyl halides at low temperatures over sodium hydroxide in a vacuum desiccator. ${ }^{2}$ Even under these conditions, certain acetylglycosyl halides decompose in a short time. ${ }^{3}$

It has now been found that the addition of a small quantity of an insoluble substance capable of neutralizing the acid formed by incipient decomposition stabilizes the acetylglycosyl halides to a striking degree. Finely powdered carbonates, especially those of calcium and barium, are particularly useful; the quantity added for stabilization is not critical, but may be varied to suit conditions of storage and purity. The solid carbonate is advantageous during the purification of the substance as well as in the final product. For some purposes the presence of the inorganic carbonate is not objectionable; but if the pure acetylglycosyl halide is desired, it can be separated when required by dissolving the material in an organic solvent and filtering the solution. ${ }^{4}$

\footnotetext{
${ }^{1}$ H. S. Isbell and H. L. Frush, J. Research, NBS 43, 161 (1949).

2 D. H. Brauns, J. Am. Chem. Soc. 51, 1820 (1929).

${ }^{3}$ In general, the reactivity of the acetylglycosyl halides increases from the fluorides to the iodides. For synthetic purposes, the chlorides and bromides are the most useful.
}

To illustrate the effectiveness of carbonates in the stabilization of acetylglycosyl halides, two $0.2-\mathrm{g}$ samples of tetraacetylmannosyl bromide, one of which contained 1 percent of calcium carbonate, were sealed in glass and stored at $20^{\circ} \mathrm{C}$. Figure 1 shows the difference in appearance after 1 week. The samples were then dissolved in $40 \mathrm{ml}$ each of chloroform, and light

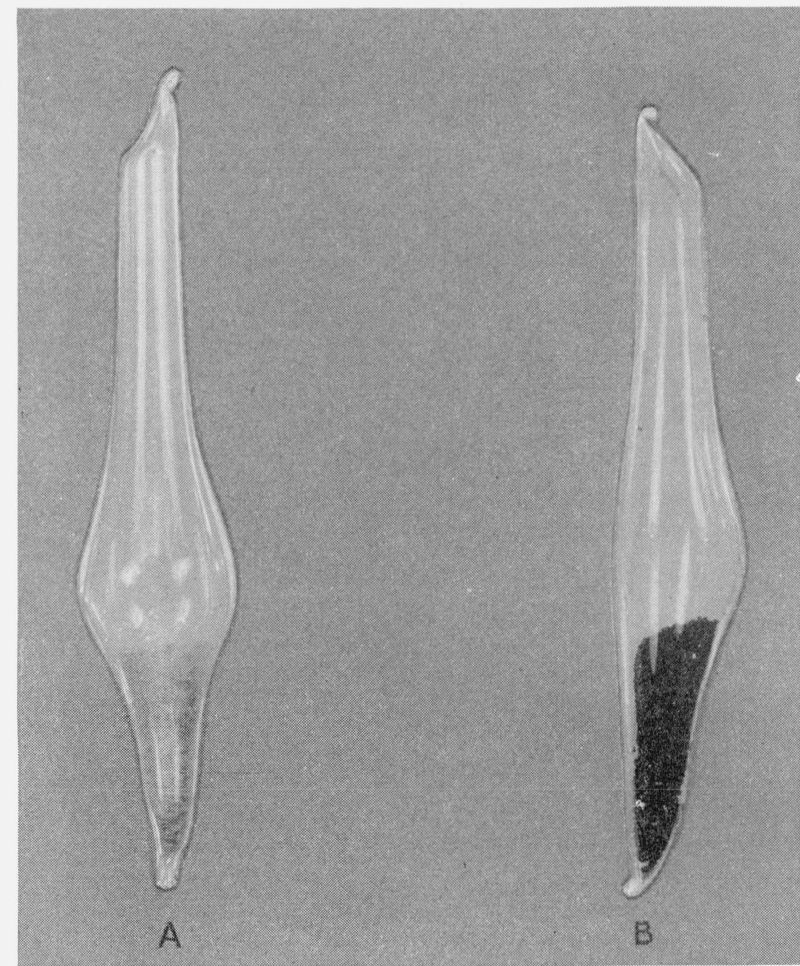

Figure 1. Effect of a trace of calcium carbonate on the stability of tetraacetylmannosyl bromide.

A, Tetraacetylmannosyl bromide containing 1 percent of calcium carbonate; $\mathrm{B}$, tetraacetylmannosyl bromide untreated.

${ }^{4}$ In some organic solvents a trace of calcium halide will be brought into solution at this point, if ealcium carbonate has been used. If this is objectionable, the acetylglycosyl halide may be recrystallized and separated immediately before use. 
transmittancy measurements were made at $436 \mu$. In a 1-cm cell, the stabilized sample showed a transmittancy of 96 percent, and the untreated sample of only 53 percent.

Calcium carbonate has been found useful, also, in the long-term storage of sugar acetates. Most of these acetates decompose to some extent on long standing, especially if slightly impure. Thus a bottle of commercial glucose pentaacetate that had been kept for several years was found to have a strong odor of acetic acid. A slightly impure heptose acetate was converted almost entirely to the free sugar during long-term storage. It has been the practice in this laboratory for the past 2 years to mix acetates, unless highly purified and carefully dried and sealed, with a small amount of calcium carbonate before storage. No such preparation has shown evidence of decomposition.

Washington, October 4, 1949. 